\title{
FIXED POINTS IN PRODUCTS OF ORDERED SPACES
}

\author{
HASKELL COHEN
}

A topological space is said to have the fixed point property if every map (i.e. continuous function) from the space to itself leaves at least one point fixed. There is a rather well known conjecture (see e.g. [3]) asking if the fact that $X$ and $Y$ have the fixed point property implies that the product space $X \times Y$ has the property. This note contains an affirmative answer in the case that $X$ and $Y$ are compact ordered sets (with the order topology, of course). It is perhaps worth noting that there is an analogous result due to Ginsburg [1] for similarity transformations on ordered sets with the product ordered lexicographically. Since a compact ordered space has the fixed point property if and only if it is connected, we may rephrase our result to read as follows. If $X$ and $Y$ are compact connected ordered spaces and $Z=X \times Y$, then $Z$ has the fixed point property. We first establish several auxiliary properties of the space $Z$.

LemMA 1. $Z$ is unicoherent (i.e. if $Z$ is the union of two closed and connected sets, then the intersection of the two sets is connected).

Proof. Suppose $Z=A \cup B$ with $A$ and $B$ closed and connected, but $A \cap B$ is not connected. Then $A \cap B=C \cup D$ with $C$ and $D$ non-null, closed, and disjoint. Since $Z$ is normal, we have disjoint open sets $P$ and $Q$ with $C \subset P$ and $D \subset Q$. For each $(x, y)$ in $Z$ we can find an open "rectangular" neighborhood $N(x, y)$ such that

$$
\begin{aligned}
& \text { if }(x, y) \text { is in } Z-A, N(x, y) \text { is in } Z-A \text {, } \\
& \text { if }(x, y) \text { is in } Z-B, N(x, y) \text { is in } Z-B, \\
& \text { if }(x, y) \text { is in } C, N(x, y) \text { is in } P \text {, and } \\
& \text { if }(x, y) \text { is in } D, N(x, y) \text { is in } Q .
\end{aligned}
$$

Since $Z$ is compact, a finite set $\mathscr{X}$ of these neighborhoods cover $Z$. We can easily find finite sets $\left\{x_{0}, x_{1}, \cdots, x_{n}\right\}$ and $\left\{y_{0}, y_{1}, \cdots, y_{m}\right\}$ in $X$ and $Y$ respectively such that

$$
\begin{aligned}
& x_{0}=\inf \{x \mid x \in X\}<x_{1}<x_{2}<\cdots<x_{n}=\sup \{x \mid x \in X\}, \\
& y_{0}=\inf \{y \mid y \in Y\}<y_{1}<y_{2}<\cdots<y_{m}=\sup \{y \mid y \in Y\},
\end{aligned}
$$

and for each $i$ and $j, R_{i j}$ is contained in some $N \in \Re$, where $R_{i j}$ $=\left\{x \mid x_{i} \leqq x \leqq x_{i+1}\right\} \times\left\{y \mid y_{j} \leqq y \leqq y_{j+1}\right\}$.

Presented to the Society, November 18, 1955; received by the editors January 21, 1955 and, in revised form, July 20, 1955. 
Now let $E=\cup\left\{R_{i j} \mid R_{i j} \cap A \neq \square\right.$ (the null set) $\}$ and $F=\bigcup\left\{R_{i j} \mid R_{i j}\right.$ $\cap B \neq \square\}$. Clearly $(E \cap F) \subset(P \cup Q)$ and $Z=E \cup F$. By means of Urysohn's Lemma (see e.g. 1.11, p. 71 [4]) we can construct a map $f$ on $X$ to $I$ such that $f\left[x_{i}, x_{i+1}\right]=[i / n,(i+1) / n]$ and a map $g$ on $Y$ to $I$ with $g\left[y_{j}, y_{j+1}\right]=[j / m,(j+1) / m]$ (where $[a, b]$ stands for the closed interval from $a$ to $b$ and $I=[0,1])$. Define $h: Z \rightarrow I^{2}$ by $h(x, y)=(f(x), g(y))$.

The following properties can be verified for $h$.

(a) $h(E) \cup h(F)=h(E \cup F)=h(Z)=I^{2}$.

(b) $A$ and $B$ connected imply that $E$ and $F$ are connected, and these in turn imply that $h(E)$ and $h(F)$ are connected.

(c) $h(E)$ and $h(F)$ are closed.

(d) $h(E) \cap h(F)=h(E \cap F)=h([E \cap F \cap P] \cup[E \cap F \cap Q])=h(E \cap F$ $\cap P) \cup h(E \cap F \cap Q)$, the union of two non-null closed disjoint sets. Note that statements (a)-(d) are a contradiction to the well known fact that $I^{2}$ is unicoherent (see e.g. Corollary, p. 118 [2]); so that the lemma is proved.

Corollary. If $C$ is a connected subset of $Z$, and if $Z-C$ is connected, then $F(C)$, (the boundary of $C$ ), is also connected.

To see that the corollary is true merely take $\bar{C}$ and $\overline{Z-C}$ as $A$ and $B$ in the lemma.

Lemma 2. If $A$ is a closed subset of $Z$, then either (a) there is a component $B$ of $Z-A$ such that $\pi_{Y}(B)=Y$ or (b) there is a component $K$ of $A$ such that $\pi_{X}(K)=X$ (where $\pi_{X}$ and $\pi_{Y}$ are the projection maps on $X$ and $Y$ ).

Proof. It is easily seen that $Z$ is locally connected; hence, components of open sets are open, and $B=\{B \mid B$ is a component of $Z-A\}$ is a collection of open sets. We prove the lemma by showing that if (a) is not true, then (b) must hold. Suppose therefore that $\pi_{Y}(B) \neq Y$ for all $B$ in $B$. We divide the proof into two parts.

CASE I. There is a $B$ with the property that $\pi_{X}(B)=X$. Since $\pi_{Y}(B) \neq Y$, there is a $y \in Y$ such that $(X \times\{y\}) \subset(Z-B)$. Since $X \times\{y\}$ is connected, it is contained in some $C$, a component of $Z-B$. Let $D=Z-C$. Clearly $D$ is connected so that by the corollary we have that $F(C)$ is connected. Since $\pi_{X}(B)=\pi_{X}(C)=X$, it is easily seen that $\pi_{X} F(C)=X$. As another consequence of local connectedness we have $F(C) \subset F(B)$ which is a subset of $A$. Since $F(C)$ is connected it is contained in a component $K$ of $A$ and $\pi_{X}(K)=X$ as was to be shown.

CASE II. For all $B \in Q$ we have $\pi_{X} B \neq X$. For each $B$ let 
$\tilde{B}=B \cup \cup\left\{C \mid C\right.$ is a component of $Z-B$ and $\left.\pi_{X} C \neq X\right\}$. If $y \notin \pi_{Y} B$, then $X \times\{y\} \subset Z-B$ and $C$ the component which contains $X \times\{y\}$ must satisfy $\pi_{X} C=X$; hence $C \llbracket \widetilde{B}$ and $y \notin \pi_{Y} \widetilde{B}$. Thus we have shown

(i) $\pi_{Y} \tilde{B}=\pi_{Y} B$.

Since $B$ is not separated from any components of its complement, we have

(ii) $\widetilde{B}$ is connected.

Since $\pi_{X} B \neq X$, there can be only one component $C$ of the complement of $B$ with $\pi_{X} C=X$; hence $Z-\tilde{B}$ consists of a single component of $Z-B$ and as a consequence

(iii) $Z-\widetilde{B}$ is connected, and

(iv) $\tilde{B}$ is open.

Now suppose $\tilde{B}_{1} \cap \tilde{B}_{2} \neq \square$. Then, using the notation $C_{i}$ is a component of $Z-B_{i}$, since $B_{1} \cap B_{2}=\square$, we have $B_{1}$ meets some $C_{2}, B_{2}$ meets some $C_{1}$, or some $C_{1}$ meets some $C_{2}$.

$(\alpha)$ If (say) $B_{1} \cap C_{2} \neq \square$, then since $B_{1}$ is connected and $B_{1} \subset Z-B_{2}$, we have $B_{1} \subset C_{2}$. If, then, $C_{1} \cap B_{2} \neq \square$, as above we show $B_{2} \subset C_{1}$ and using (i) we can write

$$
\pi_{Y} B_{1} \subset \pi_{Y}\left(C_{2}\right) \subset \pi_{Y}\left(B_{2}\right) \subset \pi_{Y}\left(C_{1}\right) \subset \pi_{Y}\left(B_{1}\right) .
$$

But since $\pi_{Y}$ is both an open and closed map, it is easily seen that all of the inclusions are proper. This contradiction shows that $C_{1} \cap B_{2}$ $=\square$, so that since $C_{1} \cup B_{1}$ is connected, we have $\left(C_{1} \cup B_{1}\right) \subset C_{2}$, and hence $\widetilde{B}_{1} \subset C_{2} \subset \widetilde{B}_{2}$.

(B) If $C_{1} \cap C_{2} \neq \square$, and $B_{1} \cap C_{2}=\square$, then $B_{1} \cap\left(C_{2} \cup B_{2}\right)=\square$ and since $C_{2} \cup B_{2}$ is connected $\left(C_{2} \cup B_{2}\right) \subset C_{1}$ and $(\alpha)$ applies; so that we have verified

(v) $\widetilde{B}_{1} \cap \widetilde{B}_{2} \neq \square$ implies $\tilde{B}_{1} \subset \widetilde{B}_{2}$ or $\tilde{B}_{2} \subset \widetilde{B}_{1}$.

Now if $B_{\lambda}$ is any member of $B$, we define

$$
B_{\lambda}^{*}=U\left\{\tilde{B} \mid \tilde{B} \supset B_{\lambda}\right\} \text {. }
$$

We have immediately that each $B^{*}$ is open and connected. In addition each $Z-B^{*}$ is connected since it is the intersection of a nest of closed connected sets so that using the corollary gives

(vi) Each $F\left(B^{*}\right)$ is connected.

Let $\tilde{A}=Z-\cup\{\tilde{B} \mid B \in \mathbb{B}\}=Z-\cup\left\{B^{*} \mid B \in \mathbb{Q}\right\}$. For each $x \in X$, $\{x\} \times Y$ is a compact connected set, and by use of (i), (iv), and (v) we can show $\{x\} \times Y \nsubseteq \cup\{\tilde{B} \mid B \in \mathbb{B}\}$; i.e.

(vii) $\pi_{X} \tilde{A}=X$.

If $\tilde{A}=M \cup N$, with $M$ and $N$ closed and disjoint, let $M^{\prime}$ $=M \cup \cup\left\{B^{*} \mid F\left(B^{*}\right) \cap M \neq \square\right\}$ and $N^{\prime}=N \cup \cup\left\{B^{*} \mid F\left(B^{*}\right) \cap N \neq \square\right\}$. It is easy to see that $M^{\prime} \cup N^{\prime}=Z$ and (vi) insures that $M^{\prime} \cap N^{\prime}$ is null. 
To show $M^{\prime}$ is open, given any $z$ in $M^{\prime}$ we must exhibit an open set in $M^{\prime}$ containing $z$. If $z$ is in one of the $B^{* \prime}$, that $B^{*}$ will do. If $z \in M$, there is an open connected set $P$ containing $z$ but missing $N$. If for some $B^{*}, P \cap B^{*} \neq \square$, then since $P \subset B^{*}$ implies $z \in B^{*}$ which is impossible, we have $P \cap F\left(B^{*}\right)$ is not null. This means, since $P \subset(Z-N)$ and (vi) holds, that $B^{*} \subset M^{\prime}$ and hence $P \subset M^{\prime}$, completing the proof. A similar argument shows $N^{\prime}$ is open; hence, since $Z$ is connected, $M$ or $N$ is null and

(viii) $\tilde{A}$ is connected.

Since $\tilde{A} \subset A$, (vii) and (viii) show there is a component $K$ of $A$ such that $\pi_{X}(K)=X$.

THEOREM. $Z$ has the fixed point property.

Proof. Let $f$ be any map on $Z$ to $Z$. Let $A=\left\{(x, y) \mid \pi_{Y} f(x, y)=y\right\}$. Clearly $A$ is closed, hence, by Lemma 2 we have either $B$, a component of $Z-A$, with $\pi_{Y}(B)=Y$, or $K$, a component of $A$, with $\pi_{X}(K)=X$. The first of these possibilities is untenable, however, since $B \cap\left\{(x, y) \mid \pi_{Y} f(x, y)>y\right\}$ and $B \cap\left\{(x, y) \mid \pi_{Y} f(x, y)<y\right\}$ are two nonnull disjoint open sets whose union is $B$, a connected set. Hence $K$ exists and since $K$ is connected, either one of the open (in $K$ ) and disjoint sets $K \cap\left\{(x, y) \mid \pi_{x} f(x, y)>x\right\}$ and $K \cap\left\{(x, y) \mid \pi_{X} f(x, y)<x\right\}$ is null, or their union cannot be all of $K$. In either case there is at least one point $\left(x^{\prime}, y^{\prime}\right)$ in $K$ such that $\pi_{x} f\left(x^{\prime}, y^{\prime}\right)=x^{\prime}$. But since $K$ is a subset of $A$, we have $\pi_{Y} f\left(x^{\prime}, y^{\prime}\right)=y^{\prime}$ and $\left(x^{\prime}, y^{\prime}\right)$ is a fixed point.

\section{REFERENCES}

1. S. Ginsburg, Fixed points of products and ordered sums of simply ordered sets, Proc. Amer. Math. Soc. vol. 5 (1954) pp. 554-565.

2. M. H. A. Newman, Elements of the topology of plane sets of points, Cambridge University Press, 1951.

3. W. L. Strother, On an open question concerning fixed points, Proc. Amer. Math. Soc. vol. 4 (1953) pp. 988-993.

4. R. L. Wilder, Topology of manifolds, Amer. Math. Soc. Colloquium Publications, vol. 32, New York, 1949.

UNIVERSITY OF TENNESSEE 